Pacific Journal of Mathematics

DERIVATIVES OF BLASCHKE PRODUCTS 


\section{DERIVATIVES OF BLASCHKE PRODUCTS}

\section{HoNG OH KIM}

Suppose $B(z)$ is an infinite Blaschke product with zeros $\left\{z_{k}\right\}$. It is known that $B^{\prime} \notin A^{2,0}$ (or $D^{1 / 2} B \notin H^{2}$ ). We extend this to get $B^{\prime} \notin$ $A^{p, p-2}(p>1)$ (or $\left.D^{\beta} B \notin H^{1 / \beta}, \beta>0\right)$ and apply this to the Taylor coefficients of an infinite Blaschke product. We also present extended versions of the Hardy-Littlewood theorem on fractional integrals and the Hardy-Littlewood embedding theorem with simple proofs. These extensions show that the above theorem becomes stronger as $p \uparrow \infty$ (or $\beta \downarrow 0$, respectively). Finally, we give sufficient conditions on $\left\{z_{k}\right\}$ in order that $D^{\beta} B \in A^{p, \alpha}$ or $\in H^{p}$, which shows that the above result is best possible in a certain sense.

Introduction. Let $U$ denote the open unit disc in the complex plane. The Hardy space $H^{p}(0<p \leq \infty)$ consists of all functions holomorphic in $U$ for which

$$
\|f\|_{p}= \begin{cases}\sup _{0 \leq r<1}\left(\int_{0}^{2 \pi}\left|f\left(r e^{i \theta}\right)\right|^{p} \frac{d \theta}{2 \pi}\right)^{1 / p} & (0<p<\infty) \\ \sup _{z \in U}|f(z)| & (p=\infty)\end{cases}
$$

is finite. The weighted Bergman space $A^{p, \alpha}$ with $0<p<\infty$ and $\alpha>-1$ consists of all functions holomorphic in $U$ for which

$$
\|f\|_{p, \alpha}^{p}=\int_{0}^{1} \int_{0}^{2 \pi}\left|f\left(r e^{\imath \theta}\right)\right|^{p}(1-r)^{\alpha} d \theta d r
$$

is finite. See [1], [15]. The Hardy space $H^{p}(0<p<\infty)$ can be considered as the "limiting space" of $A^{p, \alpha}$ as $\alpha \downarrow-1$.

If $\left\{z_{k}\right\}$ is a sequence (finite or infinite) of complex numbers in $U$ for which $\Sigma\left(1-\left|z_{k}\right|\right)<\infty$, then the Blaschke product

$$
B(z)=\prod_{k} \frac{\left|z_{k}\right|}{z_{k}} \frac{z_{k}-z}{1-\bar{z}_{k} z}
$$

converges uniformly on the compact subsets of $U$ and has $\left\{z_{k}\right\}$ as its zero set. See [5].

It is a well-known fact that a Blaschke product $B$ is a finite Blaschke product if its Dirichlet integral

$$
\frac{1}{\pi} \int_{0}^{1} \int_{0}^{2 \pi}\left|f\left(r e^{i \theta}\right)\right|^{2} r d r d \theta=\sum k\left|a_{k}\right|^{2}
$$


is finite; that is, if $B^{\prime} \in A^{2,0}$ or equivalently $\sum k^{1 / 2} a_{k} z^{k} \in H^{2}$. In $\S 1$, we extend this fact in two directions; in Theorem 1.1 we replace the condition $B^{\prime} \in A^{2,0}$ by the condition $B^{\prime} \in A^{p, p-2}$ for some $p>1$ and in Corollary 1.6 we replace the condition $\sum k^{1 / 2} a_{k} z^{k} \in H^{2}$ by the condition $\sum k^{\beta} a_{k} z^{k}$ $\in H^{1 / \beta}$. The latter is a consequence of the former but gives a new information about Taylor coefficients (Corollary 1.8). Theorem 1.1 is seen to be stronger as $p \nearrow \infty$ by means of an extension of the Hardy-Littlewood embedding theorem (due to P. R. Ahern, unpublished) which we present here as Theorem B. In $\S 2$, we extend the Hardy-Littlewood theorem on fractional integrals with a simple proof (Theorem 2.1). This extension shows that Corollary 1.6 is stronger as $\beta \searrow 0$ and gives some information about Bloch functions (Corollary 2.5). Finally, there are several known conditions on the distribution of zeros of a Blaschke product that imply that the derivative of that Blaschke product belongs to some $H^{p}$ or $A^{p, \alpha}$. See [3], [4], [13]. In $\S 3$, we extend these results to the fractional derivatives of arbitrary order of a Blaschke product (Theorem 3.1, Theorem 3.2). These theorems show that in Theorem 1.1 (or in Corollary 1.6), $A^{p, \alpha}$ (or $H^{p}$ ) cannot be replaced by a larger $A^{p-\varepsilon, \alpha}$ or $A^{p, \alpha+\varepsilon}$ (or $H^{p-\varepsilon}$ respectively) for any $\varepsilon>0$. The extended version of the Hardy-Littlewood theorem on fractional integrals is essential to prove Theorem 3.2.

Throughout this paper, $B$ always denotes a Blaschke product. $C$ denotes a constant and $C(\cdots)$ a positive constant depending only on the arguments $(\cdots)$. Both $C$ and $C(\cdots)$ may vary from occurrence to occurrence even in the proof of the same theorem.

This paper represents a part of the author's thesis written in the University of Wisconsin. The author appreciates the encouragement of his thesis advisor, Patrick R. Ahern.

\section{Finite Blaschke products.}

1.1 TheOREM. If $B^{\prime} \in A^{p, p-2}$ for some $p>1$, then $B$ is a fintie Blaschke product.

To prove this theorem, we need to quote a recent theorme of Ahern [2].

TheOrem A. Let $0<1+\alpha<p<\infty$. Then $B^{\prime} \in A^{p, \alpha}$ if and only if

$$
\int_{0}^{1}(1-r)^{\alpha} \int_{0}^{2 \pi}\left(\frac{1-B\left(r e^{i \theta}\right) \mid}{1-r}\right)^{p} d \theta d r<\infty
$$


1.2. Proof of Theorem. Suppose that $B$ is an infinite Blaschke product and let

$$
B(z)=\prod_{k=1}^{\infty} \frac{\left|z_{k}\right|}{z_{k}} \frac{z_{k}-z}{1-\bar{z}_{k} z} .
$$

Let $0<\varepsilon<1$ be fixed. Since

$$
|B(z)| \leq\left|\frac{z_{k}-z}{1-\bar{z}_{k} z}\right| \leq \frac{\left|z_{k}-z\right|}{1-\left|z_{k}\right|},
$$

$|B(z)|<\varepsilon$ if $\left|z_{k}-z\right|<\varepsilon\left(1-\left|z_{k}\right|\right)$. Let $z_{k}=r_{k} e^{i \theta_{k}}$ and $z=r e^{i \theta}$. Then

$$
\left|z_{k}-z\right|^{2}=\left(r-r_{k}\right)^{2}+4 r_{k} r \sin ^{2}\left(\frac{\theta-\theta_{k}}{2}\right) \leq\left(r-r_{k}\right)^{2}+\left(\theta-\theta_{k}\right)^{2} .
$$

If $r_{k} \leq r<r_{k}+\varepsilon\left(1-r_{k}\right) / 2$, then

$$
\left|z_{k}-z\right|^{2} \leq \frac{\varepsilon^{2}\left(1-r_{k}\right)^{2}}{4}+\left(\theta-\theta_{k}\right)^{2}
$$

so $\left|z_{k}-z\right|<\varepsilon\left(1-r_{k}\right)$ if

$$
\frac{\varepsilon^{2}\left(1-r_{k}\right)^{2}}{4}+\left(\theta-\theta_{k}\right)^{2}<\varepsilon\left(1-r_{k}\right)^{2} .
$$

Hence $|B(z)|<\varepsilon$ if $r_{k} \leq r<r_{k}+\varepsilon\left(1-r_{k}\right) / 2$ and

$$
\left|\theta-\theta_{k}\right|<\frac{\sqrt{3}}{2} \varepsilon\left(1-r_{k}\right) / 2 \text {. }
$$

Thus if $r_{k} \leq r<r_{k}+\varepsilon\left(1-r_{k}\right) / 2$, then

$$
\int_{0}^{2 \pi}\left(1-\left|B\left(r e^{\imath \theta}\right)\right|\right)^{p} d \theta \geq(1-\varepsilon)^{p} \sqrt{3} \varepsilon\left(1-r_{k}\right) .
$$

Hence

$$
\begin{aligned}
\int_{r_{k}}^{r_{k}+\varepsilon\left(1-r_{k}\right) / 2} \int_{0}^{2 \pi}\left(\frac{1-\left|B\left(r e^{i \theta}\right)\right|}{1-r}\right)^{p}(1-r)^{p-2} d \theta d r \\
\quad \geq \sqrt{3} \varepsilon(1-\varepsilon)^{p}\left(1-r_{k}\right) \int_{r_{k}}^{r_{k}+\varepsilon\left(1-r_{k}\right) / 2}(1-r)^{-2} d r \\
\quad=\frac{\sqrt{3} \varepsilon^{2}(1-\varepsilon)^{p}}{2-\varepsilon} .
\end{aligned}
$$

Now, we take an infinite subsequence $\left\{z_{k_{j}}\right\}$ of $\left\{z_{k}\right\}$ such that

$$
\left|z_{k_{\jmath}}\right|+\varepsilon\left(1-\left|z_{k_{\jmath}}\right|\right) / 2<\left|z_{k_{\jmath}+1}\right|, \quad j=1,2, \ldots
$$


We form a subproduct $B_{1}(z)$ of $B(z)$ with zero sequence $\left\{z_{k_{j}}\right\}$. Then since $|B(z)| \leq\left|B_{1}(z)\right|$, we have

$$
\begin{aligned}
\int_{0}^{1} \int_{0}^{2 \pi} & \left(\frac{1-\left|B\left(r e^{\imath \theta}\right)\right|}{1-r}\right)^{p}(1-r)^{p-2} d \theta d r \\
& \geq \int_{0}^{1} \int_{0}^{2 \pi}\left(\frac{1-\left|B_{1}\left(r e^{\imath \theta}\right)\right|}{1-r}\right)^{p}(1-r)^{p-2} d \theta d r \\
& \geq \sum_{J=1}^{\infty} \int_{r_{k_{j}}}^{r_{k_{j}}+\varepsilon\left(1-r_{k_{j}}\right) / 2} \int_{0}^{2 \pi}\left(\frac{1-\left|B_{1}\left(r e^{\imath \theta}\right)\right|}{1-r}\right)^{p}(1-r)^{p-2} d \theta d r \\
& \geq \sum_{J=1}^{\infty} \frac{\sqrt{3} \varepsilon^{2}(1-\varepsilon)^{p}}{2-\varepsilon}=\infty .
\end{aligned}
$$

So $B^{\prime} \notin A^{p, p-2}$ by Theorem $\mathrm{A}$, which is a contradiction. Hence $B$ is a finite Blaschke product.

1.3. Remarks (1) If $p=1$ and $A^{1,-1}$ is replaced by $H^{1}$, then $B$ is continuous up to the boundary by Theorem 3.11 [5]; so it is a finite Blaschke product, as is well known.

(2) To show that Theorem 1.1 really is a better result than the classical one, we present an unpublished result of Ahern which is also used again in the sequel.

THEOREM B. (Ahern). Suppose that $f(z)=O(1-|z|)^{-\gamma}$ and let $0<p$ $<q<\infty$. Then

(1) if $f \in H^{p}$, then $f \in A^{q,-1+\gamma(q-p)}$;

(2) if $f \in A^{p, \alpha}$, then $f \in A^{q, \alpha+\gamma(q-p)}$.

Proof. (Due to Ahern.) Let $M(1, \theta)=\sup _{0 \leq t<1}\left|f\left(t e^{i \theta}\right)\right|$. From the hypothesis, $\left|f\left(r e^{i \theta}\right)\right|<K(1-r)^{-\gamma}$ for some $K>0$. Then

$$
\begin{aligned}
& \int_{0}^{1}\left|f\left(r e^{i \theta}\right)\right|^{q}(1-r)^{\gamma(q-p)-1} d r \\
& \quad \leq\left|K^{q} \int_{0}^{\lambda}(1-r)^{-\gamma p-1} d r\right|+M(1, \theta)^{q} \int_{\lambda}^{1}(1-r)^{\gamma(q-p)-1} d r \\
& \quad \leq K^{q} \cdot \frac{(1-\lambda)^{-\gamma p}}{\gamma p}+M(1, \theta)^{q} \cdot \frac{(1-\lambda)^{\gamma(q-p)}}{\gamma(q-p)} .
\end{aligned}
$$


Now, let $\lambda=0$ if $M(1, \theta) \leq K$ and let $\lambda=1-(K / M(1, \theta))^{1 / \gamma}$ if $K<$ $M(1, \theta)$. Then we have for any $\theta$

$$
\begin{aligned}
& \int_{0}^{1}\left|f\left(r e^{\imath \theta}\right)\right|^{q}(1-r)^{\gamma(q-p)-1} d r \\
& \quad \leq C(K, \gamma, p, q)+C(K, \gamma, p, q) M(1, \theta)^{p} .
\end{aligned}
$$

We integrate both sides with respect to $\theta$ and use the complex maximal theorem to get (1). (2) is similarly obtained.

If $f \in H^{\infty}$, then $f^{\prime}(z)=O(1-|z|)^{-1}$; so $f^{\prime} \in A^{p, p-2}$ implies that $f^{\prime} \in A^{q, q-2}$ if $p<q$. Hence Theorem 1.1 is stronger as $p$ goes to $\infty$.

1.4. Fractional integrals and derivatives. Let $f(z)=\sum a_{k} z^{k}$ and let $\beta$ be a real number. Flett [7] defines the fractional integral of $f$ of order $\beta$ as $I^{\beta} f(z)=\Sigma(k+1)^{-\beta} a_{k} z^{k}$. If $\beta>0$, the following formula is easily verified and will be useful later:

$$
I^{\beta} f(z)=\frac{1}{\Gamma(\beta)} \int_{0}^{1}\left(\log \frac{1}{t}\right)^{\beta-1} f(t z) d t .
$$

The fractional derivative $D^{\beta} f$ of $f$ of order $\beta>0$ is defined as $D^{\beta} f=I^{-\beta} f$. The following easily verified remarks will be used without explicit mention.

1.5. REMARKS. (1) If $n$ is an integer $\geq 1$, then

$$
D^{n} f(z)=\left(\frac{d}{d z} \cdot z\right)^{n} f(z)=\sum_{k=0}^{n-1} M_{k}(z) f^{(k)}(z)+z^{n} f^{(n)}(z),
$$

where $f^{(k)}$ is the ordinary $k$ th derivative of $f$ and $M_{k}(z)$ is a monomial in $z, k=0,1, \ldots, n-1$.

(2) If $f(z)=O(1-|z|)^{-\gamma}(\gamma \geq 0)$, then

(a) $I^{\beta} f(z)=O(1-|z|)^{-(\gamma-\beta)}$, where $0 \leq \beta<\gamma$, and

(b) $D^{\beta} f(z)=O(1-|z|)^{-(\gamma+\beta)}$, where $\beta \geq 0$.

(3) For any positive integer $n$ and any positive number $p$, there exists $C_{1}, C_{2}>0$ such that

$$
C_{1} \leq \int_{0}^{2 \pi}\left|D^{n} f\left(r e^{i \theta}\right)\right|^{p} d \theta / \int_{0}^{2 \pi}\left|f^{(n)}\left(r e^{i \theta}\right)\right|^{p} d \theta \leq C_{2} .
$$

The second inequality is obvious from (1). For the first inequality, note that

$$
f(z)=\int_{0}^{1} D^{1} f(t z) d t
$$


So,

$$
\left|f\left(r e^{i \theta}\right)\right| \leq \sup _{0 \leq t \leq 1}\left|D^{1} f\left(t r e^{i \theta}\right)\right|
$$

so by the complex maximal theorem,

$$
\int_{0}^{2 \pi}\left|f\left(r e^{i \theta}\right)\right|^{p} d \theta \leq C(p) \int_{0}^{2 \pi}\left|D^{1} f\left(r e^{i \theta}\right)\right|^{p} d \theta .
$$

Since $f^{\prime}(z)=z^{-1}\left\{D^{1} f(z)-f(z)\right\}$, we get

$$
\int_{0}^{2 \pi}\left|f^{\prime}\left(r e^{i \theta}\right)\right|^{p} d \theta \leq C(p) \int_{0}^{2 \pi}\left|D^{1} f\left(r e^{i \theta}\right)\right|^{p} d \theta .
$$

We can prove (3) for any $n$ using induction, which we omit. Due to this remark we use $D^{n} f$ and $f^{(n)}$ interchangeably in this paper.

We quote some known theorems for later use. The following Theorem $\mathrm{C}$ was first proved by Hardy and Littlewood in [8, Theorem 10] when $p>1$ with slightly different definitions of the fractional drivatives and integrals.

Theorem C. (Hardy and Littlewood, Flett [7, Theorem 6].) Let $f$ be a holomorphic function in $U, 0<p<\infty$, and let $\beta>0$. Then

(1) if $f \in A^{p, \alpha}$, then $D^{\beta} f \in A^{p, \alpha+\beta p}(\alpha>-1)$;

(2) if $f \in A^{p, \alpha}$, then $I^{\beta} f \in A^{p, \alpha-\beta p}(\alpha-\beta p>-1)$.

Theorem D. (Littlewood and Paley [10, Theorems 5, 6].) Let $f$ be a holomorphic function in $U$. Then

(1) if $f^{\prime} \in A^{p, p-1}$, then $f \in H^{p}(0<p \leq 2)$;

(2) if $f \in H^{p}$, then $f^{\prime} \in A^{p, p-1}(2 \leq p<\infty)$.

We combine Theorems C and D to get the following which is more convenient for our applications.

TheOREM $\mathrm{D}^{\prime}$. ( Littlewood and Paley.) Let $f$ be a holomorphic function in $U, \beta>0$ and let $0<p<\infty$. Then

(1) if $f \in A^{p,-1+\beta p}$, then $I^{\beta} f \in H^{p}(0<p \leq 2)$;

(2) if $f \in H^{p}$, then $D^{\beta} f \in A^{p,-1+\beta p}(2 \leq p<\infty)$.

Now we can state and prove

1.6. Corollary. If $D^{\beta} B \in H^{1 / \beta}$ for some $\beta>0$, then $B$ is a finite Blaschke product. 
Proof. If $\beta>\frac{1}{2}$, then $D^{\beta} B \in H^{1 / \beta}$ implies that

$$
D^{\beta} B \in A^{2,-1+\beta(2-1 / \beta)}=A^{2,2 \beta-2},
$$

by Theorem B (1), since $D^{\beta} B(z)=O(1-|z|)^{-\beta}$. So

$$
D^{1} B=I^{\beta-1} D^{\beta} B \in A^{2,2 \beta-2-2(\beta-1)}=A^{2,0}
$$

by Theorem $\mathrm{C}$ (2); so $B$ is a finite Blaschke product by Theorem 1.1.

Next, if $\beta \leq \frac{1}{2}$, then $D^{\beta} B \in H^{1 / \beta}$ implies

$$
D^{1} B=D^{1-\beta} D^{\beta} B \in A^{1 / \beta,-1+(1-\beta) / \beta}=A^{1 / \beta, 1 / \beta-2},
$$

by Theorem $\mathrm{D}^{\prime}(2)$; so $B$ is a finite Blaschke product by Theorem 1.1.

1.7. Remark. Since $D^{\beta} B(z)=O(1-|z|)^{-\beta}$, Theorem 2.1 in the next section shows that $D^{\beta} B \in H^{1 / \beta}$ implies that $D^{\gamma} B \in H^{1 / \gamma}$ if $0<\gamma<\beta$. This means that Corollary 1.6 becomes stronger a $\beta \searrow 0$.

1.8. Corollary. If $B(z)=\sum a_{k} z^{k}$ and $\sum(k+1)^{p-1}\left|a_{k}\right|^{p}<\infty$ for some $p \geq 1$, then $B$ is a fintie Blaschke product.

Proof. Suppose that $B$ is an infinite Blaschke product. Then for any $\beta>0, D^{\beta} B(z)=\Sigma(k+1)^{\beta} a_{k} z^{k} \notin H^{1 / \beta}$ by Corollary 1.6. First we apply the Hausdorff-Young inequality (Duren [5, Theorem 6.1]) with $q=1 / \beta$ $\geq 2$ and $1 / p+1 / q=1$, and get

$$
\infty=\left\|D^{\beta} B\right\|_{p}^{p} \leq \sum(k+1)^{\beta p}\left|a_{k}\right|^{p} ;
$$

so $\Sigma(k+1)^{p-1}\left|a_{k}\right|^{p}=\infty$ for $1 \leq p \leq 2$ since $\beta p=p-1$.

Next we apply the Hardy-Littlewood inequality (Duren [5, Theorem 6.2]) with $q=1 / \beta \geq 2$ and get

$$
\begin{aligned}
\infty & =\left\|D^{\beta} B\right\|_{q}^{q} \leq C(q) \sum(k+1)^{q-2}\left|(k+1)^{\beta} a_{k}\right|^{q} \\
& =C(q) \sum(k+1)^{q-1}\left|a_{k}\right|^{q} .
\end{aligned}
$$

This completes the proof.

1.9. Remark. The conditions $\Sigma(k+1)^{p-1}\left|a_{k}\right|^{p}<\infty$ for a sequence $\left\{a_{k}\right\}$ which is bounded by 1 are seen to be independent, for any two different values of $p$. For it $1 \leq s<t$, take

$$
a_{k}= \begin{cases}2^{(1 / t-1) \nu} & \text { if } k=2^{\nu}, \nu>0 \text { integer } \\ 0 & \text { otherwise }\end{cases}
$$


and $b_{k}=(k+1)^{-1}\{\log (k+1)\}^{-1 / s}, k=1,2, \ldots$; we can easily check that

$$
\sum(k+1)^{s-1}\left|a_{k}\right|^{s}<\infty \text { but } \sum(k+1)^{t-1}\left|a_{k}\right|^{t}=\infty
$$

and

$$
\sum(k+1)^{s-1}\left|b_{k}\right|=\infty \text { but } \sum(k+1)^{t-1}\left|b_{k}\right|^{t}<\infty .
$$

Note also that $a_{k}$ 's and $b_{k}$ 's are all bounded by one.

\section{Hardy-Littlewood theorem on fractional integrals.}

2.1 THEOREM. If $f \in H^{p}$ and $f(z)=O(1-|z|)^{-\gamma}$ with $0<\gamma \leq 1 / p$, then $I^{\beta} f \in H^{q}$ with $q=\gamma p /(\gamma-\beta)$ where $0<\beta<\gamma$.

Proof. We first assume that $0<\beta<1$. From the hypothesis, $|f(t z)| \leq$ $K(1-t|z|)^{-\gamma} \leq K(1-t)^{-\gamma}$ for some constant $K>0$. Set $M(r, \theta)=$ $\sup _{0 \leq t<1}\left|f\left(t r e^{i \theta}\right)\right|$. Since $1-t \leq \log 1 / t,(\log 1 / t)^{\beta-1} \leq(1-t)^{\beta-1}$. So

$$
\begin{aligned}
\left|I^{\beta} f(z)\right| & \leq \frac{1}{\Gamma(\beta)} \int_{0}^{1}\left(\log \frac{1}{t}\right)^{\beta-1}|f(t z)| d t \\
& \leq \frac{1}{\Gamma(\beta)} \int_{0}^{1}(1-t)^{\beta-1}|f(t z)| d t .
\end{aligned}
$$

Now, we proceed as in the proof of Theorem B and get

$$
\int_{0}^{1}(1-t)^{\beta-1}\left|f\left(t r e^{i \theta}\right)\right| d t \leq C(K, \beta, \gamma)+C(K, \beta, \gamma) M(r, \theta)^{(\gamma-\beta) / \gamma}
$$

So,

$$
\begin{aligned}
\int_{0}^{2 \pi}\left|I^{\beta} f\left(r e^{i \theta}\right)\right|^{q} d \theta & \leq C(K, \beta, \gamma, p)+C(K, \beta, \gamma, p) \int_{0}^{2 \pi} M(r, \theta)^{p} d \theta \\
& \leq C(K, \beta, \gamma, p)+C(K, \beta, \gamma, p) \int_{0}^{2 \pi}\left|f\left(r e^{i \theta}\right)\right|^{p} d \theta .
\end{aligned}
$$

We used the complex maximal theorem in the last inequality. So $f \in H^{p}$ implies that $I^{\beta} f \in H^{q}$ where $q=\gamma p /(\gamma-\beta)$.

Next, we note that $I^{\beta} f(z)=O(1-|z|)^{-\gamma+\beta}$. If $\beta \geq 1$, we write $\beta=\beta_{1}+\beta_{2}+\cdots+\beta_{m}$ with $0<\beta_{1}, \beta_{2}, \ldots, \beta_{m}<1$. Then successive application of the above argument with $\beta_{1}, \beta_{2}, \ldots, \beta_{m}$ proves the theorem.

2.2 REMARK. If $f \in H^{p}$, then it is known that $f(z)=O(1-|z|)^{-1 / p}$; $I^{\beta} f \in H^{q}$ where $q=1 / p \cdot p /(1 / p-\beta)=p /(1-\beta p)$. This is the wellknown theorem of Hardy and Littlewood on fractional integrals, [9, Theorem 30], [5, Theorem 5.12]. 
2.3 CoRollary. If $f \in A^{p, \alpha}$ and $f(z)=O(1-|z|)^{-\gamma}$ where $0<p \leq 2$, $\alpha>-1$ and $(\alpha+1) / p<\gamma \leq(\alpha+2) / p$, then $I^{\beta} f \in H^{q}$ where $q=$ $(\gamma p-\alpha-1) /(\gamma-\beta)$ and $(\alpha+1) / p \leq \beta<\gamma$.

Proof. Since $p \leq 2, \quad I^{(\alpha+1) / p} f \in H^{p}$ by Theorem $\mathrm{D}^{\prime}$ (1). Note $I^{(\alpha+1) / p} f(z)=O(1-|z|)^{-(\gamma-(\alpha+1) / p)}$. By Theorem 2.1, $I^{\beta} f=$ $I^{\beta-(\alpha+1) / p} I^{(\alpha+1) / p} f \in H^{q}$, where $q=(\gamma p-\alpha-1) /(\gamma-\beta)$.

2.4. REMARK. If $f \in A^{p, \alpha}$, then $f(z)=O(1-|z|)^{-(\alpha+2) / p}[15$, Corollary B]; so $I^{\beta} f \in H^{q}$ with $q=p /(\alpha+2-\beta p)$ where $(\alpha+1) / p \leq$ $\beta<(\alpha+2) / p$ if $0<p \leq 2$.

A function $f$ holomorphic in $U$ is said to be a Bloch function if $f^{\prime}(z)=O(1-|z|)^{-1}$. See Pommerenke [12] for more about Bloch functions.

2.5 COROLlaRY. If $f$ is a Bloch function with $f^{\prime} \in A^{p, \alpha}$ where $p>\alpha+$ $1>0$, then $f \in H^{q}$ for all $q<\infty$.

Proof. Let $0<p \leq 2$. Since $D^{1} f(z)=O(1-|z|)^{-1}$, for any sufficiently small $\beta>0, D^{\beta} f=I^{1-\beta} D^{1} f \in H^{(p-\alpha-1) / \beta}$ by Corollary 2.3 ; in particular, $f \in H^{(p-\alpha-1) / \beta}$ for any $\beta>0$. Let $2<p<\infty$. We can easily check that $f^{\prime} \in A^{p, \alpha}$ implies that $f^{\prime} \in A^{2, \delta}$ if $\delta>2(\alpha+1) / p-1$. But since $2(\alpha+1) / p<2$, we can choose $\delta$ so that $\delta+1<2$. Then $f \in H^{q}$ for all $q<\infty$ as above.

2.6. Remarks. (1) If $f$ is a Bloch function, then $f^{\prime} \in A^{p, \alpha}$ when $p<\alpha+1$.

(2) If $f$ is a Bloch function and $f^{\prime} \in H^{p}(0<p<1)$, then $f^{\prime} \in A^{2,1-p}$ by Theorem B (1); so $f \in H^{q}$ for all $q<\infty$ by Corollary 2.5.

(3) The example $f(z)=\log (1-z)$ shows that $q=\infty$ cannot be allowed in the conclusion of Corollary 2.5.

3. Blaschke products. Throughout this section, let $B(z)$ be a Blaschke product with zeros $\left\{z_{k}\right\}$. We write $d_{k}=1-\left|z_{k}\right|$ as usual.

3.1. TheOREM. Let $\beta>0,0<p<\infty$ and let $\alpha>-1$. Then (1) if $\sum d_{k}^{\alpha+2-\beta p}<\infty$, then

$$
D^{\beta} B \in A^{p, \alpha} \quad\left(\max \left(\frac{\alpha+2}{\beta+1}, \frac{\alpha+1}{\beta}\right)<p<\frac{\alpha+2}{\beta}\right) ;
$$


(2) if $\sum d_{k}^{p} \log 1 / d_{k}<\infty$, then

$$
D^{\beta} B \in A^{p, \alpha} \quad\left(p=\frac{\alpha+2}{\beta+1} \geq \frac{\alpha+1}{\beta}\right) ;
$$

(3) if $\sum d_{k}^{\delta}<\infty$ for some $\delta<(\alpha+2) /(\beta+1)$, then

$$
D^{\beta} B \in A^{p, \alpha} \text { for any } p<\frac{\alpha+2}{\beta+1}
$$

$((\alpha+2) /(\beta+1) \geq(\alpha+1) / \beta$ i.e., $\beta \geq \alpha+1)$.

3.2. THEOREM. Let $\beta>0$. Then

(1) if $\sum d_{k}^{1-\beta p}<\infty$, then

$$
D^{\beta} B \in H^{p} \quad\left(\frac{1}{\beta+1}<p<\frac{1}{\beta}\right) ;
$$

(2) if $\sum d_{k}^{p} \log 1 / d_{k}<\infty$, then

$$
D^{\beta} B \in H^{p} \quad\left(p=\frac{1}{\beta+1}\right)
$$

(3) if $\sum d_{k}^{\delta}<\infty$ for some $\delta<1 /(\beta+1)$, then $D^{\beta} B \in H^{p}$ for any $p<1 /(\beta+1)$.

3.3 Remarks. (1) If $\beta=1$ in Theorem 3.1, it is the result of Protas (for $A^{1, \alpha}$, see [13, Theorem 1]) and of Ahern (for $1 \leq p<\infty$, see [1, Theorems 4.2, 5.1]).

(2) If $\beta=1$ in Theorem 3.2, it is the result of Protas [13, Theorems $2,3]$.

(3) Theorem 3.1 (1) with $\beta=1$ and $1 \leq p \leq 2$ and Theorem 3.2 (1) with $\beta=1$ were shown to have their "converses". See Ahern [1, Theorems 4.2, 6.2]. Both Theorems 3.1 (1) and 3.2 (1) for $0<p \leq 2$ can be seen to have their converses with the same method of proof. For example, if $D^{\beta} B \in H^{p}(1 /(\beta+1)<p<1 / \beta)$, then $D^{\beta} B \in A^{2,-1+\beta(2-p)}$ by Theorem B (1); so by Theorem C (2)

$$
D^{1} B=I^{\beta-1} D^{\beta} B \in A^{2,-1+\beta(2-p)-2(\beta-1)}=A^{2,1-\beta p} ;
$$

so

$$
\sum \frac{k^{2}\left|a_{k}\right|^{2}}{k^{2-\beta p}}=\sum k^{\beta p}\left|a_{k}\right|^{2}<\infty
$$

where $B(z)=\sum a_{k} z^{k}$. Now, by the theorem of Carleson quotd by Ahern on page 335 of [1], there exists a set $E$ of capacity 0 such that $\sum d_{k}(c)^{1-\beta p}$ $<\infty$ if $c \in U \backslash E$ and $d_{k}(c)=1-\left|z_{k}(c)\right|$ where $z_{k}(c)$ are the zeros of 
$(c-B(z)) /(1-\bar{c} B(z))$. It would be interesting to know whether the converse holds in general.

(4) Theorem 3.1(2) with $\beta=1$ connects one of Rudin's results:

$$
\text { if } \sum d_{k} \log 1 / d_{k}<\infty, \quad \text { then } B^{\prime} \in A^{1,0}
$$

[14, Theorem I], and one of Protas' results:

$$
\text { if } \sum d_{k}^{1 / 2} \log 1 / d_{k}<\infty, \text { then } B^{\prime} \in H^{1 / 2} \quad\left(=A^{1 / 2,-1}\right)
$$

[13, Theorem 3].

The following computational lemmas are well known.

Lemma E (Tsuji $[\mathbf{1 7}, p .226])$. If $|a| \leq 1$ and $0<\rho<1$, then

$$
\int_{0}^{2 \pi} \frac{d \theta}{\left|1-a \rho e^{i \theta}\right|^{\gamma}} \leq \begin{cases}C(\gamma) \frac{1}{(1-|a| \rho)^{\gamma-1}} & \text { if } \gamma>1 ; \\ C \log \frac{1}{1-|a| \rho} & \text { if } \gamma=1 ; \\ C & \text { if } \gamma<1 .\end{cases}
$$

Lemma F (Shields and Williams $[16$, Lemma 6]). If $0<r, \rho<1$, then

$$
\int_{0}^{1} \frac{(1-r)^{\alpha}}{(1-r \rho)^{\gamma}} d r \leq \begin{cases}C(\alpha, \gamma)(1-\rho)^{1+\alpha-\gamma} & \text { if } \gamma>1+\alpha>0 \\ C(\alpha) \log \frac{1}{1-\rho} & \text { if } \gamma=1+\alpha>0 \\ C & \text { if } \gamma<1+\alpha>0 .\end{cases}
$$

3.4. LEMMA. $B^{(n)}(z)$ is a finite sum of the terms of the form

$$
\begin{aligned}
\sum C & \left(\begin{array}{c}
\kappa, \lambda, \ldots, \mu \\
k, l \cdots m
\end{array}\right) B_{k, l, \ldots, m}(z) \\
& \times \frac{1-\left|z_{k}\right|^{2}}{\left(1-\bar{z}_{k} z\right)^{\kappa}} \frac{1-\left|z_{l}\right|^{2}}{\left(1-\bar{z}_{l} z\right)^{\lambda}} \cdots \frac{1-\left|z_{m}\right|^{2}}{\left(1-\bar{z}_{m} z\right)^{\mu}}
\end{aligned}
$$

where $\sum$ is the sum over $k, l \neq k, \ldots, m \neq k, l, \ldots$,

$$
C\left(\begin{array}{l}
k, \lambda, \ldots, \mu \\
k, l, \ldots, m
\end{array}\right) \leq C(n)<\infty \quad \text { for any } k, l, \ldots, m ; \kappa, \lambda, \ldots, \mu,
$$

$$
B_{k, l, \ldots, m}(z)=B(z) \frac{1-\bar{z}_{k} z}{z_{k}-z} \cdot \frac{1-\bar{z}_{l} z}{z_{l}-z} \cdots \frac{1-\bar{z}_{m} z}{z_{m}-z},
$$

$$
(\kappa-1)+(\lambda-1)+\cdots+(\mu-1)=n .
$$


Proof. We proceed by induction. It is true for $n=1$, since

$$
B^{\prime}(z)=\sum_{k} B_{k}(z) \frac{1-\left|z_{k}\right|^{2}}{\left(1-\bar{z}_{k} z\right)^{2}} .
$$

See Protas [13] for example. Assume that the lemma is true for $n \geq 1$. Consider $B^{(n+1)}(z)$. It is a finite sum of derivatives of terms like (1), i.e.,

$$
\begin{aligned}
\sum C & \left(\begin{array}{l}
\kappa, \lambda, \ldots, \mu \\
k, l, \ldots, m
\end{array}\right) B_{k, l, \ldots, m}^{\prime}(z) \\
& \times \frac{1-\left|z_{k}\right|^{2}}{\left(1-\bar{z}_{k} z\right)^{\kappa}} \cdot \frac{1-\left|z_{l}\right|^{2}}{\left(1-\bar{z}_{l} z\right)^{\lambda}} \cdots \frac{1-\left|z_{m}\right|^{2}}{\left(1-\bar{z}_{m} z\right)^{\mu}} \\
& +\sum C\left(\begin{array}{l}
\kappa, \lambda, \ldots, \mu \\
k, l, \ldots, m
\end{array}\right) B_{k, l, \ldots, m}(z) \\
& \times \frac{\kappa z_{k}\left(1-\left|z_{k}\right|^{2}\right)}{\left(1-\bar{z}_{k} z\right)^{\kappa+1}} \cdot \frac{1-\left|z_{l}\right|^{2}}{\left(1-\bar{z}_{l} z\right)^{\lambda}} \cdots \frac{1-\left|z_{m}\right|^{2}}{\left(1-\bar{z}_{m} z\right)^{\mu}}
\end{aligned}
$$

+ similar terms to the second term.

But since

$$
B_{k, l, \ldots, m}^{\prime}(z)=\sum_{s \neq k, l, \ldots, m} B_{k, l, \ldots, m, s}(z) \frac{1-\left|z_{s}\right|^{2}}{\left(1-\bar{z}_{s} z\right)^{2}}
$$

the first term above is of the form (1) with $n+1$ in place of $n$ in (2) and (4). The other terms are also of the form (1) with $n+1$ in place of $n$ by absorbing the constants like $\kappa \bar{z}_{k}$ in $C\left(\begin{array}{c}\alpha+1, \lambda, \ldots, \mu \\ k, l, \ldots, m\end{array}\right)$. This completes the proof.

3.5. Proof of Theorem 3.1. By Theorem C, it suffices to prove the theorem with $\beta$ replaced by its integral part $n$. We may also consider the ordinary $n$th derivative $B^{(n)}$ instead of $D^{n} B$ because of 1.5. (3). We only prove (1). (2) and (3) can be read off in the proof of (1). Suppose first that

$$
\max \left(\frac{\alpha+2}{n+1}, \frac{\alpha+1}{n}\right)<p \leq \frac{1}{n} .
$$

By Lemma $3.4,\left|B^{(n)}\left(r e^{i \theta}\right)\right|$ is dominated by a finite sum of the terms of the form

$$
C(n) \sum_{m} \cdots \sum_{l} \sum_{k} \frac{1-\left|z_{k}\right|}{\left|1-\bar{z}_{k} r e^{i \theta}\right|^{\kappa}} \cdot \frac{1-\left|z_{l}\right|}{\left|1-\bar{z}_{l} r e^{i \theta}\right|^{\lambda}} \cdots \frac{1-\left|z_{m}\right|}{\left|1-\bar{z}_{m} r e^{i \theta}\right|^{\mu}}
$$


$(k, l, \ldots, m$ runs through all positive integers here), which is

$$
C(n)\left(\sum_{k} \frac{1-\left|z_{k}\right|}{\left|1-\bar{z}_{k} r e^{i \theta}\right|^{\kappa}}\right) \cdot\left(\sum_{l} \frac{1-\left|z_{l}\right|}{\mid 1-\bar{z}_{r} r e^{i \theta} \mu^{\mu}}\right) \cdots\left(\sum_{m} \frac{1-\left|z_{m}\right|}{\mid 1-\bar{z}_{m} r e^{\imath \theta} \mu^{\mu}}\right) .
$$

Since $0<p \leq 1, \int_{0}^{2 \pi}\left|B^{(n)}\left(r e^{i \theta}\right)\right|^{p} d \theta$ is dominated by a finite sum of the integrals of the form

$$
\begin{array}{r}
C(n, p) \int_{0}^{2 \pi}\left(\sum_{k} \frac{1-\left|z_{k}\right|}{\left|1-\bar{z}_{k} r e^{i \theta}\right|^{\kappa}}\right)^{p} \cdot\left(\sum_{l} \frac{1-\left|z_{l}\right|}{\left|1-\bar{z}_{l} r e^{i \theta}\right|^{\mu}}\right)^{p} \\
\cdots\left(\sum_{m} \frac{1-\left|z_{m}\right|}{\mid 1-\bar{z}_{m} r e^{i \theta} \mu}\right)^{p} d \theta .
\end{array}
$$

Now, we apply the Hölder's inequality with indices

$$
\frac{\kappa-1}{n}+\frac{\lambda-1}{n}+\cdots+\frac{\mu-1}{n}=1 .
$$

Then the integral is domainated by

$$
\begin{gathered}
\left(\int_{0}^{2 \pi}\left(\sum_{k} \frac{1-\left|z_{k}\right|}{\left|1-\bar{z}_{k} r e^{i \theta}\right|^{\kappa}}\right)^{n p /(\kappa-1)} d \theta\right)^{(\kappa-1) / n} \\
\quad \ldots\left(\int_{0}^{2 \pi}\left(\sum_{m} \frac{1-\left|z_{m}\right|}{\left|1-\bar{z}_{m} r e^{i \theta}\right|}\right)^{n p /(\mu-1)} d \theta\right)^{(\mu-1) / n}
\end{gathered}
$$

Since $n p /(\kappa-1) \leq n p \leq 1$, the integral of the first factor is dominated by

$$
\sum_{k} \int_{0}^{2 \pi} \frac{\left(1-\left|z_{k}\right|\right)^{n p /(\kappa-1)}}{\left|1-z_{k} r e^{i \theta}\right|^{\kappa n p /(\kappa-1)}} d \theta
$$

which is

$$
\begin{aligned}
& \leq C(n, p, \kappa) \sum_{k} \frac{\left(1-\left|z_{k}\right|\right)^{n p /(\kappa-1)}}{\left(1-\left|z_{k}\right| r\right)^{\kappa n p /(\kappa-1)-1}} \\
& \leq C(n, p, \kappa) \sum_{k} \frac{\left(1-\left|z_{k}\right|\right)^{p}}{\left(1-\left|z_{k}\right| r\right)^{(n+1) p-1}} .
\end{aligned}
$$

We used Lemma E with $\kappa n p /(\kappa-1)>\kappa n(\alpha+2) /(\kappa-1)(n+1)>1$ in the first inequality and replaced some power of $\left(1-\left|z_{k}\right| r\right)$ by the same power of the smaller $\left(1-\left|z_{k}\right|\right)$ to get the second inequality. We estimate 
the other factors similarly and replace the constants $C(n, p, \kappa), \ldots$ by the bigger constant $C(n, p)$ and get

$$
\int_{0}^{2 \pi}\left|B^{(n)}\left(r e^{i \theta}\right)\right|^{p} d \theta \leq C(n, p) \sum_{k} \frac{\left(1-\left|z_{k}\right|\right)^{p}}{\left(1-\left|z_{k}\right| r\right)^{(n+1) p-1}} .
$$

Now, we integrate both sides with respect to $(1-r)^{\alpha} d r$ and use Lemma F to get

$$
\int_{0}^{1} \int_{0}^{2 \pi}\left|B^{(n)}\left(r e^{i \theta}\right)\right|^{p}(1-r)^{\alpha} d \theta d r \leq C(n, p) \sum_{k}\left(1-\left|z_{k}\right|\right)^{\alpha+2-n p} .
$$

So we get $B^{(n)} \in A^{p, \alpha}$ if $\sum d_{k}^{\alpha+2-n p}<\infty$.

Before proving the remaining case, we note that the above for $n=1$ and the results of Ahern [1, Theorems 4.2, 5.1] and Theorem B completely prove the theorem for $n=1$. Now assume $1 / n<p<(\alpha+2) / n$. We proceed by induction. As noted above the theorem is true for $n=1$. Let $n \geq 2$ and assume that (1) is true for $n-1$. Since we can easily check that $\alpha+2-n p=(\alpha-p)+2-(n-1) p$,

$$
\frac{(\alpha-p)+2}{n}<p, \quad \frac{(\alpha-p)+1}{n-1}<p \quad \text { and } \quad \frac{(\alpha-p)+2}{n-1}>p,
$$

we have $B^{(n-1)} \in A^{p, \alpha-p}$ by the induction hypothesis. By Theorem C (1), $B^{(n)} \in A^{p, \alpha}$. This completes the proof

3.6. Proof of Theorem 3.2. Here again we prove only (1). (2) and (3) can be read off from the proof of (1). Write $\beta=n-b$ where $n$ is a positive integer and $0<b \leq 1$. Assume first that $0<p \leq 2$. By Theorem $D^{\prime}(1)$

$$
D^{\beta} B=I^{b} D^{n} B \in H^{p} \quad \text { if } D^{n} B \in A^{p, b p-1} .
$$

But $D^{n} B \in A^{p, b p-1}$ by Theorem 3.1 since

$$
\sum d_{k}^{(b p-1)+2-n p}=\sum d_{k}^{1-(n-b) p}<\infty,
$$

and

$$
\max \left(\frac{(b p-1)+2}{n+1}, \frac{(b p-1)+1}{n}\right)<p<\frac{(b p-1)+2}{n}
$$

can easily be checked. Next, assume $p>1$. We use induction on the positive integer $\nu$ with $1 /(\nu+1) \leq \beta<1 / \nu$. If $\nu=1$ and $\frac{1}{2} \leq \beta<1$, then $\frac{1}{2}<\beta p<1$; so $\Sigma d_{k}^{1-\beta p}<\infty$ implies $D^{1} B \in H^{\beta p}$ by the argument above. Hence $D^{\beta} B=I^{1-\beta} D^{1} B \in H^{p}$ by Theorem 2.1. Now assume that 
we have proved the theorem for $\beta$ in $1 / \nu \leq \beta<1 /(\nu-1)$, and let $1 /(\nu+1) \leq \beta<1 / \nu$. Since

$$
\begin{gathered}
\sum d_{k}^{1-(1 / \nu)(\nu \beta p)}=\sum d_{k}^{1-\beta p}<\infty, \\
\nu \beta p>\nu \beta \geq \frac{\nu}{\nu+1}=\frac{1}{1 / \nu+1}, \text { and } \nu \beta p<\nu=\frac{1}{1 / \nu},
\end{gathered}
$$

we get $D^{1 / \nu} B \in H^{\nu \beta p}$ by the induction hypothesis. By Theorem 2.1 again

$$
D^{\beta} B=I^{1 / \nu-\beta} D^{1 / \nu} B \in H^{p} .
$$

This completes the proof.

3.7. Remark. We used Theorem 3.1 (1) to prove Theorem 3.2 (1). Conversely, we can deduce Theorem 3.1 (1) from Theorem 3.2 (1). Suppose that Theorem 3.2 (1) is proved. We shall prove Theorem 3.1 (1). Let $n \geq 1$ an integer, $\alpha>-1$ and $q>0$ be fixed so that

$$
\max \left(\frac{\alpha+2}{n+1}, \frac{\alpha+1}{n}\right)<q<\frac{\alpha+2}{n} .
$$

Assume that $\sum d_{k}^{\alpha+2-n p}<\infty$. We need to show that $B^{(n)} \in A^{p, \alpha}$. Since $q-(\alpha+2) /(n+1)>0$ and $q-(\alpha+1) / n>0$, we can choose $p>0$ very close to $q$ so that

(i) $q-(\alpha+2) /(n+1)>(q-p) /(n+1)>0$ and

(ii) $0<(n q-(\alpha+1)) / p<n$.

We set $\beta=(n p-(\alpha+1)) / p$. Then $1-\beta p=\alpha+2-n q$. Also $1 /(\beta+1)<p$ by (i), and $1 / \beta>p$ since $q<(\alpha+2) / n$. By Theorem 3.2 (1), $D^{\beta} B \in H^{p}$; so $D^{\beta} B \in A^{q,-1+\beta(q-p)}$ by Theorem $\mathrm{B}$ (1). Now by Theorem C (1)

$$
D^{n} B=D^{n-\beta} D^{\beta} B \in A^{q,-1+\beta(q-p)+q(n-\beta)}=A^{q, \alpha},
$$

since $-1+\beta(q-p)+q(n-\beta)=n q-\beta p-1=\alpha$. This completes the proof.

\section{REFERENCES}

[1] P. R. Ahern, The mean modulus and derivative of an inner function; Indiana University Math. J., 28, No. 2, (1979); 311-347.

[2] _ The Poisson integral of a singular measure; to appear.

[3] P. R. Ahern and D. N. Clark, On inner functions with $H^{p}$ derivative, Michigan Math. J., 21 (1974), 115-127.

[4] On inner functions with $B^{p}$ derivative, Michigan Math. J., 23 (1976), 107-118.

[5] P. L. Duren, Theory of $H^{p}$ spaces, Academic Press, New York, NY 1970. 
[6] P. L. Duren, B. W. Romberg and A. L. Shields, Linear functionals on $H^{p}$ spaces with $0<p<1$, J. Reine Angew. Math., 238 (1969), 32-60.

[7] T. M. Flett, The dual of an inequality of Hardy and Littlewood and some related inequalities, J. Math. Analysis and Appl., 38 (1972), 746-765.

[8] G. H. Hardy and J. E. Littlewood, Theorems concerning mean values of analytic or harmonic functions, Quart. J. Math., 12 (1941), 221-256.

[9] _ Some properties of fractional integrals II, Math. Z., 34 (1932), 403-439.

[10] J. E. Littlewood and R. E. A. C. Paley, Theorems on Fourier series and power series (II), Proc. London Math. Soc., (2), 42 (1937), 52-89.

[11] D. J. Newman and H. S. Shapiro, The Taylor coefficients of inner functions, Michigan Math. J., 9 (1962), 249-255.

[12] Ch. Pommerenke, On Bloch functions, J. London Math. Soc., (2), 2 (1970), 689-695.

[13] D. Protas, Blaschke product with derivative in $H^{p}$ and $B^{p}$, Michigan Math. J., 30 (1973), 393-396.

[14] W. Rudin, The radial variation of analytic functions, Duke Math. J., 22 (1955), 235-242.

[15] J. H. Shapiro, Mackey topologies, reproducing kernels, and diagonal maps on the Hardy and Bergman spaces, Duke Math. J., 43, No. 1 (1976), 187-202.

[16] A. L. Shields and D. L. Williams, Bounded projection, duality and multipliers in spaces of analytic functions, Trans. Amer. Math. Soc., 162 (1971), 287-302.

[17] M. Tsuji, Potential Theory in Modern Function Theory, Chelsea Publishing Co., New York, NY (1959).

Received September 15, 1982 and in revised form December 20, 1982.

\section{UNIVERSITY OF WISCONSIN}

MADISON, WI 53706

Current address: Kyungpook National University

Taegu, 635, Korea 


\section{PACIFIC JOURNAL OF MATHEMATICS \\ EDITORS}

DONALD BABBITt (Managing Editor)

University of California

Los Angeles, CA 90024

J. Dugundu

University of Southern California

Los Angeles, CA 90089-1113

R. FINN

Stanford University

Stanford, CA 94305

HERMANN FLASCHKA

University of Arizona

Tucson, AZ 85721
C. C. MOORE

University of California

Berkeley, CA 94720

ARTHur Ogus

University of California

Berkeley, CA 94720

Hugo RossI

University of Utah

Salt Lake City, UT 84112

H. SAMELSON

Stanford University

Stanford, CA 94305

ASSOCIATE EDITORS

R. ARENS

E. F. BECKENBACH

B. H. NeumanN (1906-1982)

F. WOLF

K. YoshidA

\section{SUPPORTING INSTITUTIONS}

UNIVERSITY OF ARIZONA

UNIVERSITY OF BRITISH COLUMBIA

UNIVERSITY OF OREGON

CALIFORNIA INSTITUTE OF TECHNOLOGY

UNIVERSITY OF CALIFORNIA

MONTANA STATE UNIVERSITY

UNIVERSITY OF SOUTHERN CALIFORNIA

UNIVERSITY OF NEVADA, RENO

STANFORD UNIVERSITY

UNIVERSITY OF HAWAII

NEW MEXICO STATE UNIVERSITY

UNIVERSITY OF TOKYO

UNIVERSITY OF UTAH

OREGON STATE UNIVERSITY

WASHINGTON STATE UNIVERSITY

UNIVERSITY OF WASHINGTON 


\section{Pacific Journal of Mathematics}

Vol. 114, No. $1 \quad$ May, 1984

David Marion Arnold and Charles Irvin Vinsonhaler, Typesets and cotypesets of rank-2 torsion free abelian groups $\ldots \ldots \ldots \ldots \ldots \ldots \ldots 1$

Duncan Alan Buell and Richard Howard Hudson, Solutions of certain

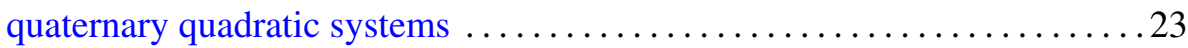

Hans Delfs and Manfred Knebusch, Separation, retractions and homotopy

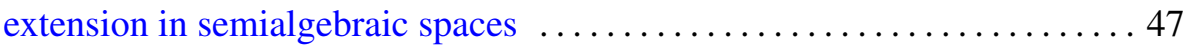

I. Erdélyi and Sheng-Wang Wang, A spectral duality theorem for closed

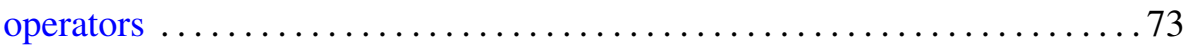

Theodore William Gamelin, Weak compactness of representing measures for $R(K)$

Kenneth R. Goodearl and T. H. Lenagan, Krull dimension of

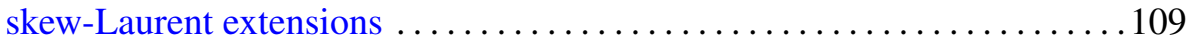

Daniel Joseph Gross, Compact quotients by $\mathbf{C}^{*}$-actions . . . . . . . . . . . 149

Goo Ishikawa, Satoshi Koike and Masahiro Shiota, Critical value sets of

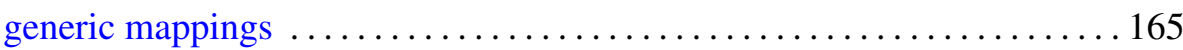

Hong Oh Kim, Derivatives of Blaschke products $\ldots \ldots \ldots \ldots \ldots \ldots \ldots \ldots \ldots$

Erhard Luft and Denis Karmen Sjerve, 3-manifolds with subgroups

$Z \oplus Z \oplus Z$ in their fundamental groups $\ldots \ldots \ldots \ldots \ldots \ldots \ldots \ldots \ldots \ldots \ldots \ldots$

George Clifford Nelson, Boolean powers, recursive models, and the Horn

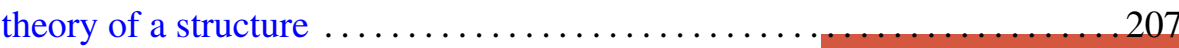

W. J. Phillips, Flow under a function and discrete decomposition of properly infinite $W^{*}$-algebras

Teodor C. Przymusiński, A solution to a problem of E. Michael ... 235

Bruce Harvey Wagner, Derivations of quasitriangular algebras 243 